\title{
Detection of non-thermal X-ray emission from the Ophiuchus cluster with INTEGRAL
}

\section{Eckert, S. Paltani* and T. J.-L. Courvoisier}

ISDC Data Centre for Astrophysics, Observatory, University of Geneva, Switzerland

E-mail: Dominique.Eckerteunige.ch

Stephane.Paltani@unige.ch

It is well-known that a significant number of galaxy clusters exhibit large-scale non-thermal activity thanks to the detection of radio synchrotron emission. However, the inverse-Compton component which is predicted to dominate over the thermal emission in hard X-rays has never been unambiguously detected so far. The Ophiuchus cluster is a massive, nearby cluster of galaxies located in the Galactic bulge region. Thanks to the very large INTEGRAL exposure time in this region, we were able to extract a high-quality spectrum in a broad energy band (3-120 keV) with IBIS/ISGRI and JEM-X. A significant excess (6.4 sigma) above $25 \mathrm{keV}$ is found compared to the extrapolation of the thermal emission, which is probably the signature of non-thermal emission. Using available radio data on the cluster, we derive a magnetic field in the range 0.1-0.2 $\mu G$.

7th INTEGRAL Workshop

Copenhagen, Denmark

September 8-11, 2008

\footnotetext{
* Speaker.
} 


\section{Introduction}

The detection of diffuse radio emission on a large scale $(\sim \mathrm{Mpc})$ in several clusters of galaxies (e.g. Coma, [1]) indicates that clusters of galaxies are particle acceleration sites. Indeed, the emission is due to synchrotron radiation from relativistic electrons, whose energy-loss timescale is much shorter than the age of the Universe [2]. To explain the presence of a large population of relativistic electrons, two main theoretical models have been developed. The electrons can be either accelerated directly through shock acceleration [3] or originate from the decay of pions produced in interactions between cosmic-ray protons and thermal ions. Inverse-Compton (IC) scattering of the relativistic electrons with the Cosmic Microwave Background (CMB) should therefore produce non-thermal emission in the hard X-ray domain. While the synchrotron radiation in the radio domain has been known for a long time, there is still no firm detection of the inverse-Compton component in the radio domain, which is crucial to measure the magnetic field strength and to discriminate between the different models which lead to the presence of relativistic particles in clusters. To the present day, this component could have been detected in the Coma [4] and Abell 2256 [5] clusters using BeppoSAX/PDS data, although these detections are still weak and controversial. Therefore, confirmation of the results by other missions is important.

The Ophiuchus cluster of galaxies $(z=0.028,[6])$ is the second brightest galaxy cluster in the $\mathrm{X}$-ray band and the most massive in our close neighbourhood. X-ray observations with the ASCA satellite [7] revealed a very hot temperature $(k T \sim 12 \mathrm{keV})$ and a complex temperature structure, which they interpret as the result of a merging event in the recent past. In the radio domain, the cluster has been associated with the steep-spectrum radio source MSH 17-203 [6], which implies the presence of a population of relativistic electrons confined in the cluster. In the hard X-ray band, the cluster was observed by BeppoSAX [8]. A possible high-energy excess compared to the thermal model was found, although the detection $(2 \sigma)$ was not statistically significant.

Thanks to its location in the galactic bulge $\left(l=9.5^{\circ}, b=0.5^{\circ}\right)$, INTEGRAL accumulated a large amount of data on the cluster since the beginning of the mission. Here we present the results of an analysis of more than $3 \mathrm{Msec}$ of data on the Ophiuchus cluster with the IBIS/ISGRI and JEM-X instruments on board INTEGRAL. For further details, see [9].

\section{Morphology}

We extracted mosaic images from both ISGRI and JEM-X data to study the morphology of the source and its energy dependence. Figure 1 shows the full ISGRI and JEM-X images in the 20-40 keV and 3-18 keV bands. For comparison with the PSF of the instruments, the insets show the image of the point source V2400 Oph, located $50 \mathrm{arcmin}$ from the cluster, re-normalized to the same brightness. In the JEM-X image, we can see that the source is clearly extended. On the other hand, the extension of the source is not obvious in the ISGRI image. 

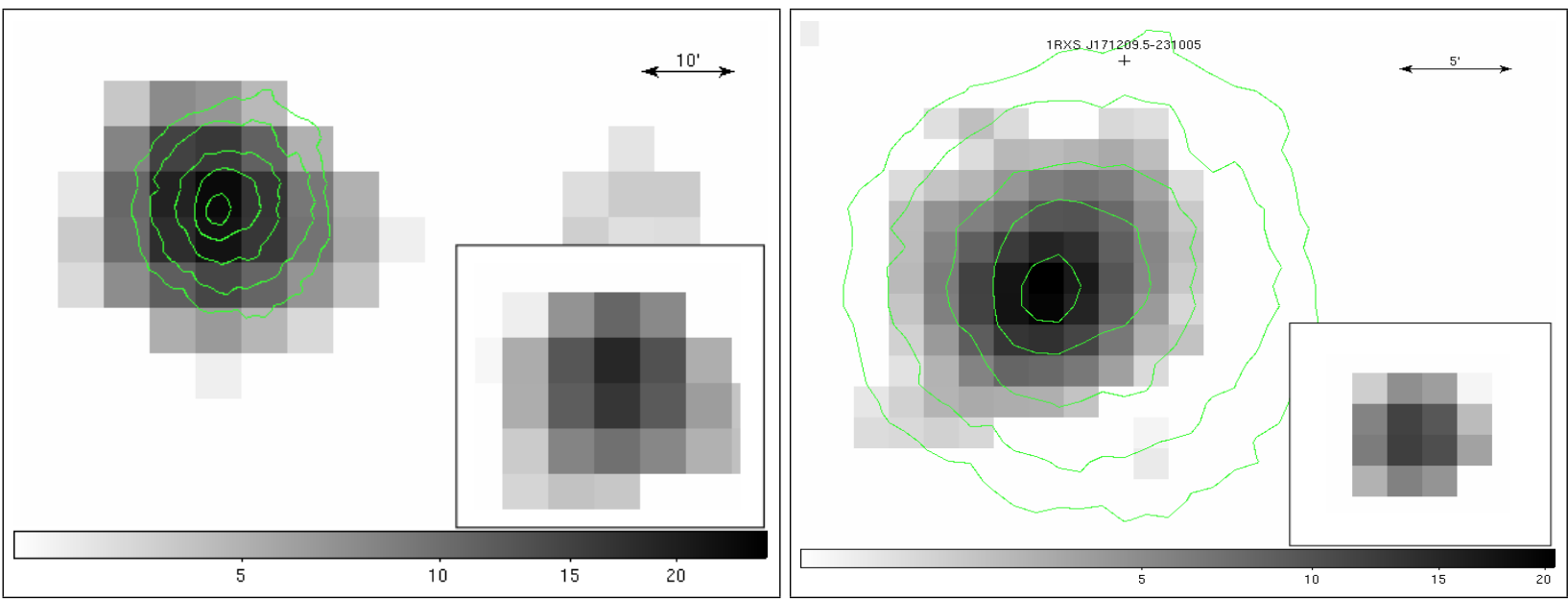

Figure 1: INTEGRAL images of the Ophiuchus cluster in the 20-40 keV band from ISGRI (left) and in the 3-18 keV band from JEM-X (right). For comparison, the insets show the image of a known point source in the same field, the Cataclysmic Variable V2400 Oph.

In order to investigate the temperature variations of the gas within the detected region, we created a 3-7/7-18 keV hardness ratio map with JEM-X. Indeed, assuming that the 3-20 keV band is completely dominated by the thermal emission, the hardness ratio can be converted into a temperature measurement. In the center of the cluster, we found a hardness ratio $H R=0.78 \pm 0.09$, which corresponds to a temperature $k T=9.1_{-0.9}^{+1.4}$. In general, no deviations from the central value are found above $0.7 \sigma$, which corresponds to temperature variations of $\pm 2 \mathrm{keV}$. This result is consistent with previous ASCA results [7]. Indeed, even though much hotter temperatures are found in the outer regions of the cluster, the core shows an almost iso-thermal profile, which is consistent with our results. This analysis shows that a single-temperature bremsstrahlung model should describe well the total JEM-X spectrum.

On the other hand, with a core radius $R_{c} \sim 3.2 \operatorname{arcmin}$, the source should be almost point-like for ISGRI. [10] presented the results of ISGRI data simulations assuming a uniform disk. For a disk of an angular size of $5 \mathrm{arcmin}$, they evaluate that the relative error on the reconstructed flux assuming that the source is point-like is $\sim 5 \%$. Since the cluster has a peaked radial profile instead of uniform disk, the relative error on the flux extracted by the standard OSA spectral extraction tool is $<5 \%$. For this reason, it is safe to treat the source as point-like for ISGRI.

Since the source is not resolved spatially, in principle we cannot exclude the possibility that a hard point source in the cluster (e.g. a very absorbed AGN) has a significant influence on the hard $\mathrm{X}$-ray spectrum. Nevertheless, to study the energy dependence of the source we can compute the best-fit position of the source in several energy bands and compare the results to the low-energy morphology. For comparison, public Chandra data on the cluster with very high angular resolution $\left(<1^{\prime \prime}\right)$ are available, which allows us to distinguish the point sources from the diffuse emission. Comparing the best-fit position found in the 20-24, 24-30 and 30-40 keV bands with the Chandra image, we find that the ISGRI positions are well-centered on the central cD galaxy, and are not con- 
sistent with any of the weak point sources detected with Chandra. This indicates that the emission detected by ISGRI is truly of diffuse origin, and that significant contamination of the high-energy spectrum by point sources is unlikely.

\section{Broad-band spectrum of the cluster}

For spectral analysis, we used the standard ISGRI OSA 7.0 spectral extraction software with a carefully-selected input source catalog, and extracted the JEM-X spectrum from the mosaic in order to take the extension of the source into account. Unlike previous versions of OSA, the calibration of ISGRI is now valid down to $17 \mathrm{keV}$. Thanks to the very large exposure time, we were able to reach a high signal-to-noise spectrum from both JEM-X (3-18 keV) and ISGRI (17-60 $\mathrm{keV})$. Thanks to the small overlap between the ISGRI and JEM-X energy ranges, we found that no cross-calibration factor between the two instruments was needed, which gives credit to the work of the instrument teams for the release of OSA version 7.0.

To analyze the resulting spectra, we used the XSPEC v11 package [11] with appropriate instrumental response files. In a first approach, we fitted the total spectrum with a single MEKAL model [12], with the redshift of the cluster fixed to $z=0.028$ [6] and the abundance fixed to 0.49 relative to the solar value [13]. The fit gives a relatively high temperature $(k T=10.8 \pm 0.3 \mathrm{keV})$ in agreement with $A S C A$ results [7], but the high reduced chi-squared $\chi_{\text {red }}^{2}=2.3$ indicates that the data are not well represented by the model. Using only the 3-20 keV part of the spectrum, we found a good fit with a significantly lower temperature, $k T=8.5 \pm 0.5 \mathrm{keV}$. Later observations of the cluster with Suzaku [14] found a temperature $k T=9.3 \pm 0.1 \mathrm{keV}$ in agreement with our measurement, and significantly lower than the ASCA value, which probably indicates that $A S C A$ was not suitable for the measurement of a high-temperature plasma.

Fig. 2 shows the total ISGRI/JEM-X spectrum and the residuals compared to the MEKAL model at $k T=8.5 \mathrm{keV}$ fitted to the $3-20 \mathrm{keV}$ part of the spectrum. Significant positive residuals are found in the high-energy part of the spectrum compared to the extrapolation of the model. This implies the presence of another spectral component in addition to the thermal bremsstrahlung emission. For instance, the bad representation of the data could be due to the presence of non-thermal hard X-ray emission. To test this possibility, we added a second component to the model, under the form of a power-law with photon index fixed to $\Gamma=2.0$, similar to [8]. The results of the fitting procedure with the addition of the second component can be found in Table 1. We can see in the table that the fit is significantly improved by the addition of the non-thermal component. In Method 1 , the temperature and normalization of the thermal component are left free while fitting, while in Method 2, these 2 parameters are fixed to the value fitted to the low-energy part of the spectrum. We can see that the temperature values obtained with the two different ways are consistent, which proves that fixing the MEKAL component to the value fitted in the $3-20 \mathrm{keV}$ band is relevant.

Using Method 2, we were also able to constrain the acceptable range for the photon index of the power-law. Leaving only the photon index and normalization of the power-law free while 


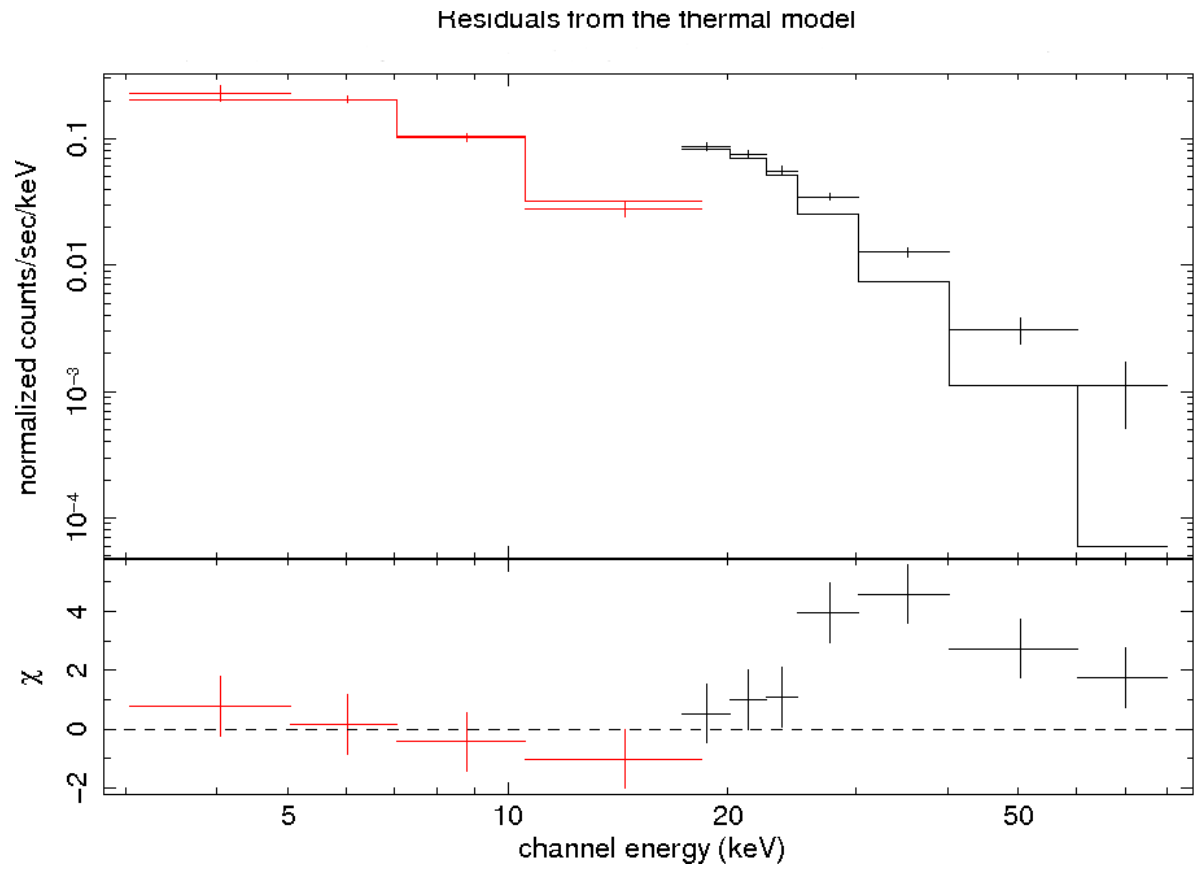

Figure 2: Combined INTEGRAL JEM-X (red) and ISGRI (black) folded spectrum of the Ophiuchus cluster. The solid line shows the model at $k T=8.5 \pm 0.4 \mathrm{keV}$ fitted to the low-energy data (3-20 keV) and extrapolated to higher energies. Ths bottom panel shows the residuals from the model. A significant excess is found above $25 \mathrm{keV}$.

\begin{tabular}{|c|c|c|c|c|c|}
\cline { 2 - 6 } \multicolumn{1}{c|}{} & $\chi_{\text {red }}^{2}$ & $P_{F-\text { test }}{ }^{a}$ & $k T[\mathrm{keV}]$ & $\operatorname{Flux}_{H X R}{ }^{b}$ & $C L_{H X R} \sigma^{c}$ \\
\hline Method 1 & 0.93 & $2 \cdot 10^{-4}$ & $8.56_{-0.35}^{+0.37}$ & $10.1 \pm 2.5$ & 4.0 \\
Method 2 & 1.05 & $7 \cdot 10^{-5}$ & $8.50_{-0.45}^{+0.48}$ & $8.2 \pm 1.3$ & 6.4 \\
\hline
\end{tabular}

Table 1: Results of the spectral fitting procedure for a thermal + power-law model, with abundance and redshift values fixed to the literature value and photon index fixed to $\Gamma=2.0$, similar to the work of [8]. In Method 1, the temperature and the normalization of the bremsstrahlung component are left free while fitting. In Method 2, we used the data in the 3-20 keV band to fix the temperature and normalization of the thermal component, and let free only the normalization of the non-thermal component. ${ }^{a} P_{F-t e s t}$ is the null hypothesis probability when adding the non-thermal component given by the F-test. ${ }^{b} 20-60 \mathrm{keV}$ flux of the non-thermal component, in units of $10^{-12} \mathrm{ergs} \mathrm{s}^{-1} \mathrm{~cm}^{-2}$. The errors are quoted at the $1 \sigma$ level. ${ }^{c}$ Confidence level for the detection of the non-thermal component. 
fitting, we found $\Gamma=1.62_{-0.35}^{+0.32}$. This is slightly harder than the photon index of 2.0 used to derive the properties of the non-thermal excess, but it is consistent with this value (within $1 \sigma$ ). With such a hard photon index, it is obvious that the excess cannot be due to the presence of a second thermal component. Indeed, if instead of a power-law we fit the excess with a second thermal component, we find an unrealistic lower limit of $k T \geq 50 \mathrm{keV}$ to the temperature of the second thermal component. This is consistent with the spectro-imaging results obtained from the JEM-X hardness ratio map, which did not reveal the presence of any specifically hot region within the core of the cluster. Therefore, we can conclude with high confidence that the high-energy excess detected by INTE$G R A L$ is non-thermal.

\section{Discussion}

In order to investigate the origin of the non-thermal component, we constructed a Spectral Energy Distribution (SED) of the non-thermal component together with existing radio data, and constructed synchrotron/inverse-Compton models as a function of the magnetic field $B$ and electron cut-off energy $E_{c u t}$. Comparing the strength of the radio and hard X-ray emissions, and using the formula

$$
\frac{P_{s y n c}}{P_{I C}}=\frac{u_{B}}{u_{\gamma}}=\frac{B^{2} / 8 \pi}{u_{C M B}}
$$

where $P_{s y n c}, P_{I C}$ is the power emitted through synchrotron, respectively IC emission, $B$ is the mean magnetic field strength and $u_{C M B}$ is the well-known energy density of the Cosmic Microwave Background, we can constrain the mean magnetic field value in the cluster. The bottom panel of Fig. 3 shows the SED models computed for different values of magnetic field $B$ and electron cut-off energy $E_{c u t}$. Based on these models, we estimate the magnetic field strength to be

$$
B \sim 0.1-0.2 \mu G
$$

This value is similar to the one derived with the same method in the Coma cluster [4]. However, this is an order of magnitude lower than the typical values $(B \gtrsim 1 \mu G)$ derived from Farady rotation measure [15].

If the magnetic field in the cluster is of the order of $\sim 1 \mu G$, the hard X-ray (HXR) emission cannot be due to IC scattering. Therefore, we also considered an alternative where the hard X-rays come from synchrotron emission from ultra-relativistic electrons ([16], [17]). In this model, we find that the HXR emission can be well-represented by a population of electrons with a cut-off energy $E \sim \mathrm{PeV}$ in a magnetic field $\sim 1-10 \mu G$. The top panel of Fig. 3 shows the resulting models. The figure clearly shows that in this case, significant emission is expected to arise in the $\mathrm{TeV}$ range, which could possibly be detected by the current generation of Cherenkov telescopes (H.E.S.S., MAGIC). 


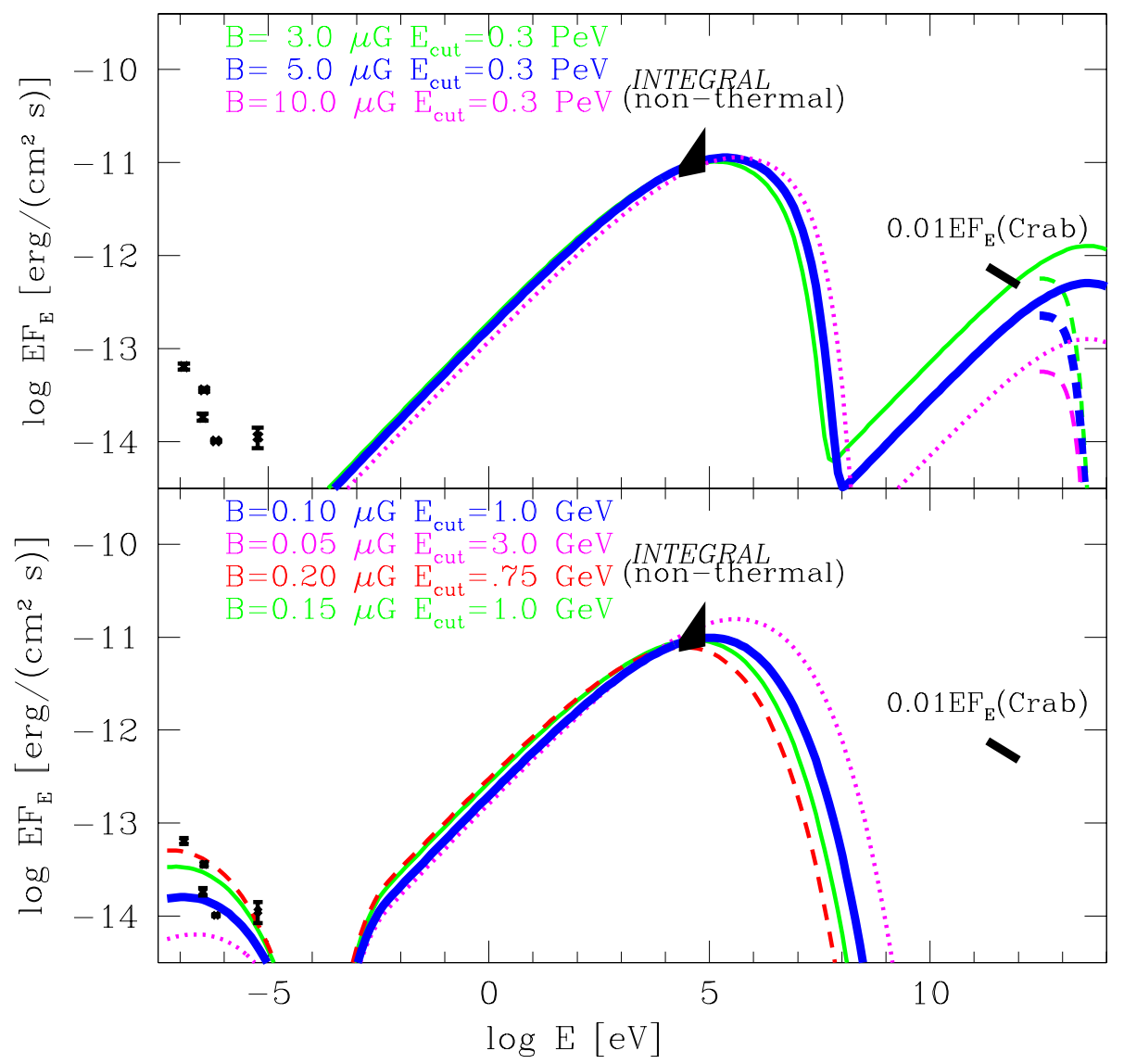

Figure 3: Models for the Spectral Energy Distribution (SED) of the non-thermal emission from the Ophiuchus cluster. The radio data are from [6]. The black triangle represents the INTEGRAL measurement of non-thermal emission (this work). In the top panel, we considered a high-energy electron population of $E \sim$ $\mathrm{PeV}$ electrons radiating in the HXR range through synchrotron emission and in the TeV range through IC scattering, while in the bottom panel, a more conventional modeling with $E \sim \mathrm{GeV}$ electrons was performed. The dashed lines above $\sim 10 \mathrm{TeV}$ shows attenuation of the spectrum by interaction of the $\gamma$-ray photons with the extragalactic infra-red and CMB radiation fields.

\section{Conclusion}

Thanks to a very long ( $3 \mathrm{Ms}$ ) observation of Ophiuchus with INTEGRAL, we were able to detect unambiguously, at a very robust statistical significance $(6.4 \sigma)$, the sign of non-thermal hard $\mathrm{X}$-ray emission. This result is extremely important, because it settles the long-standing issue of the existence or not of non-thermal hard X-ray emission at a high level in clusters of galaxies.

The origin of the high-energy emission is still unclear. In the standard inverse-Compton scenario, the low magnetic field value measured through the ratio between the power emitted through synchrotron and inverse-Compton emission is puzzling, because it falls short by 1 order of magnitude of the typical values measured through Faraday rotation. If a more standard magnetic field value is used, then the hard X-ray emission cannot be due to inverse-Compton scattering. In this case, alternative models (e.g. synchrotron emission from multi-TeV electrons) should be consid- 
ered. Regarding the origin of the high-energy electrons, recent Suzaku results [14] indicate that the cluster is basically in a relaxed state, which discards the standard primary models where electrons are directly accelerated in merging events. In this case, secondary models where the electrons are produced by proton-proton collisions [18] or dark-matter annihilation [19] are favoured. In any case, multi-wavelength information (at radio, $\mathrm{GeV}$ and $\mathrm{TeV}$ energies) is required to better constrain the spectral energy distribution of the non-thermal emission from the cluster. In particular, the Ophiuchus cluster will be a very appealing target for the Fermi/GLAST mission, which has been launched on June 10, 2008. Indeed, according to [18], Ophiuchus should be by far the brightest cluster in the $\mathrm{GeV}$ range, and should be easily detectable for Fermi. Observations of the cluster with Fermi will therefore bring a crucial input to the understanding of the population of high-energy particles in the cluster.

\section{References}

[1] L. Feretti and G. Giovannini, Galaxy Clusters in the Radio: Relativistic_Plasma and__CM/Radio _ Galaxy Interaction Processes, ArXiv Astrophysics e-prints (Mar., 2007),[astro-ph/0703494],

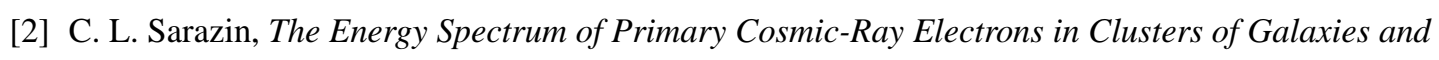
Inverse Compton Emission, ApJ 520 (Aug., 1999) 529-547,', [arXiv :astro-ph/9901061].

[3] G. Brunetti, G. Setti, L. Feretti, and G. Giovannini, Particle reacceleration in the Coma cluster: radio properties and hard X-ray emission, MNRAS 320 (Jan., 2001) 365-378,

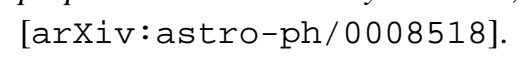

[4] R. Fusco-Femiano, D. dal Fiume, L. Feretti, G. Giovannini, P. Grandi, G. Matt, S. Molendi, and A. Santangelo, Hard X-Ray Radiation in the Coma Cluster Spectrum, ApJ L 513 (Mar., 1999) L21-L24, [a

[5] R. Fusco-Femiano, R. Landi, and M. Orlandini, Confirmation of the Presence of Nonthermal Hard X-Ray Excess in the Cluster A2256 from Two Epoch Observations, ApJ L 624 (May, 2005) L69-L72,

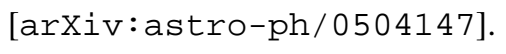

[6] M. D. Johnston, H. V. Bradt, R. E. Doxsey, F. E. Marshall, D. A. Schwartz, and B. Margon, The Ophiuchus cluster - A bright X-ray cluster of galaxies at low galactic latitude, ApJ 245 (May, 1981) 799-804.

[7] M. Watanabe, K. Yamashita, A. Furuzawa, H. Kunieda, and Y. Tawara, Temperature Map and Iron Abundance of the Ophiuchus Cluster of Galaxies, PASJ 53 (Aug., 2001) 605-610.

[8] J. Nevalainen, T. Oosterbroek, M. Bonamente, and S. Colafrancesco, Nonthermal Hard X-Ray Emission in Galaxy Clusters Observed with the BeppoSAX PDS, ApJ 608 (June, 2004) 166-178, '[arXiviastro-ph/0311142]:

[9] D. Eckert, N. Produit, S. Paltani, A. Neronov, and T. J.-L. Courvoisier, INTEGRAL discovery of non-thermal hard X-ray emission from the Ophiuchus cluster, A\&A 479 (Feb., 2008) 27-34, ['[arXiv:0712:2326]'

[10] M. Renaud, A. Gros, F. Lebrun, R. Terrier, A. Goldwurm, S. Reynolds, and E. Kalemci, Imaging extended sources with coded mask telescopes: application to the INTEGRAL IBIS/ISGRI instrument, A\&A 456 (Sept., 2006) 389-394. 
[11] K. A. Arnaud, XSPEC: The First Ten Years, in Astronomical Data Analysis Software and Systems V (G. H. Jacoby and J. Barnes, eds.), vol. 101 of Astronomical Society of the Pacific Conference Series, pp. 17-+, 1996.

[12] J. S. Kaastra and R. Mewe, Coronal Plasmas Modeling and the MEKAL code, in Atomic Data Needs for X-ray Astronomy, p. 161 (M. A. Bautista, T. R. Kallman, and A. K. Pradhan, eds.), pp. 161-+, Oct., 2000.

[13] J. J. Mohr, B. Mathiesen, and A. E. Evrard, Properties of the Intracluster Medium in an Ensemble of Nearby Galaxy Clusters, ApJ 517 (June, 1999) 627-649, [ar

[14] Y. Fujita, M. Nagai, K. Hayashida, S. Inoue, H. Matsumoto, N. Okabe, T. Reiprich, C. Sarazin, and M. Takizawa, Suzaku Observation of the Ophiuchus Galaxy Cluster: One of the Hottest Relaxed Cool Core Clusters, Submitted to PASJ (2008).

[15] C. L. Carilli and G. B. Taylor, Cluster Magnetic Fields, ARA\&A 40 (2002) 319-348, [arXiviastro-ph/0110655]',

[16] A. N. Timokhin, F. A. Aharonian, and A. Y. Neronov, On the non-thermal high energy radiation of galaxy clusters, A\&A 417 (Apr., 2004) 391-399,'[astro-ph/0305149]!

[17] S. Inoue, F. A. Aharonian, and N. Sugiyama, Hard X-Ray and Gamma-Ray Emission Induced by Ultra-High-Energy Protons in Cluster Accretion Shocks, ApJ L 628 (July, 2005) L9-L12, '[arXiviastro-ph/0505398]',

[18] C. Pfrommer, Simulating cosmic rays in clusters of galaxies - III. Non-thermal scaling relations and

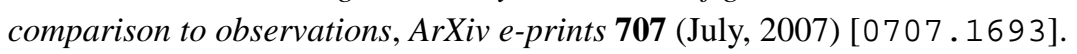

[19] S. Profumo, Non-thermal X-rays from the Ophiuchus cluster and dark matter annihilation, ArXiv 'e-prints 801 (Jan., 2008) [0801.0740]! 